
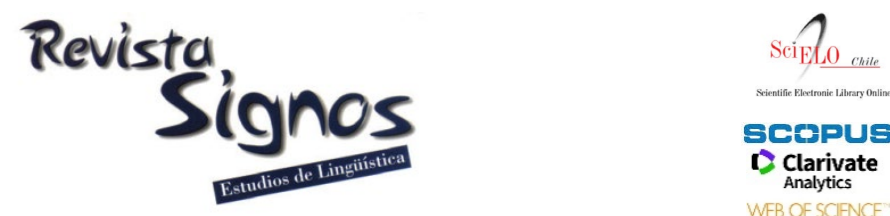

\title{
El análisis de las relaciones discursivas como recurso en el seguimiento de la Enfermedad de Alzheimer
}

\author{
Discourse relations analysis as a resource for Alqheimer's \\ disease monitoring
}

Anayeli Paulino

UNIVERSIDAD NACIONAL AUTÓNOMA DE MÉXICO

MÉXICO

apaudje@gmail.com

\section{Iria da Cunha}

UNIVERSIDAD NACIONAL DE EDUCACIÓN A DISTANCIA

ESPAÑA

iriad@flog.uned.es

\author{
Gerardo Sierra \\ UNIVERSIDAD NACIONAL AUTÓNOMA DE MÉXICO \\ MÉXICO \\ gsierram@iingen.unam.mx
}

Laura Hernández-Domínguez

ÉCOLE DU TECHNOLOGIE SUPÉRIEURE

CANADÁ

laudobla@gmail.com

Recibido: 22-XI-2018 / Aceptado: 25-IX-2019

DOI: $10.4067 /$ S0718-09342020000100242

\section{Resumen}

Este trabajo plantea una propuesta para el análisis y medición de la coherencia discursiva en el discurso conectado de adultos mayores diagnosticados con demencia de tipo Alzheimer (DTA) en contraste con adultos mayores en un proceso de envejecimiento sano. Por medio de la Teoría de la Estructura Retórica (RST), se analizan las relaciones retóricas subyacentes en la conversación de siete pacientes de la tercera edad en diferentes etapas de la demencia, y seis adultos mayores que presentaban un estado cognitivo sano. El procedimiento consistió en la segmentación en unidades semánticas de diálogo, el etiquetado de relaciones y la elaboración de diagramas arbóreos. Adicionalmente, se realiza una primera evaluación de las relaciones producidas por los cuidadores con el fin de sopesar la influencia de estos en la producción de la conversación. Encontramos que los pacientes cognitivamente sanos formulan más relaciones y de más tipos que los pacientes con demencia. Las relaciones de Causa involuntaria, Solución, Concesión, Elaboración y Resultado involuntario se asocian al discurso de adultos sanos. Por otro lado, los cuidadores utilizan ciertas relaciones retóricas con fines pragmáticos. Creemos que los resultados arrojados hacen plausible la valoración de esta propiedad discursiva dentro del catálogo de afectaciones lingüísticas que ayuden a construir el perfil lingüístico de un adulto que sufre DTA y de uno sano.

Palabras Clave: Relaciones retóricas, unidades semánticas de diálogo, coherencia, Rhetorical Structure Theory, discurso conversacional, demencia tipo Alzheimer. 


\begin{abstract}
This paper explores the results of a measurement proposal for discourse coherence of elderly patients who have been diagnosed with dementia of the Alzheimer's type (DAT), in contrast with cognitively-healthy elderly. By means of the Rhetorical Structure Theory, the rhetorical relations underlying conversations of seven elderly patients in different stages of dementia and six cognitively healthy elderly were analyzed. The procedure involved segmentation of semantic discourse units (SDUs), labeling of relations and making of discourse trees. Additionally, relations of caregivers were counted for analyzing their contributions and conversational strategies in semistructured interviews. We found that elderly patients produce more relations and use more variety of them. Involuntary Cause, Solution, Concession, Elaboration and Involuntary Result are related to cognitively healthy subjects' discourse. On the other hand, some rhetorical relations are used by caregivers for pragmatic purposes. Due to our results, we believe that it is plausible to add the discourse coherence into the catalog of linguistic affectations considered for the clinic profile of a patient with DTA.
\end{abstract}

Key Words: Rhetorical relations, semantic dialogue units, coherence, Rhetorical Structure Theory, dementia of the Alzheimer's type.

\title{
INTRODUCCIÓN
}

La demencia tipo Alzheimer (DTA), en tanto síndrome de carácter neurodegenerativo, afecta esferas cognitivas imprescindibles para el correcto desarrollo del sujeto, sean motrices (funciones ejecutivas, articulatorias) o cognitivas (afectaciones mnémicas, gnósicas, visuoespaciales, etc.) (Roa Rojas, Martínez Ruíz \& García Peña, 2017; Albert Cabrera, Martínez Pérez, Gutiérrez Ravelo, Hakim Rodríguez \& Pérez Davison, 2014). El lenguaje, un proceso biológico cuya ejecución en el habla implica la conversión de esquemas cognitivos a esquemas lingüísticos emitidos (García Meilán, Martínez-Sánchez \& Ivanova, 2018), es una de las habilidades que suele verse afectada desde etapas tempranas de la enfermedad, y durante décadas se ha visto como una vía alterna no invasiva de exploración y estudio.

Es por esto que la variedad de fenómenos en los diferentes niveles de la lengua han motivado nuevos estudios cuyo fin es conformar un catálogo exacto de las características lingüísticas más afectadas o mejor preservadas durante la DTA (Fraser, Meltzer \& Rudzicz, 2016; Asgari, Kaye \& Dodge, 2017; Hernández-Domínguez, Ratté, Sierra-Martínez \& Roche-Bergua, 2018; Toledo, Aluísio, dos Santos, Brucki, Trés, de Oliveira \& Mansur, 2018; Toth, Hoffmann, Gosztolya, Vincze, Szatloczki, Banreti \& Kálmán, 2018) para dar cuenta del impacto de la demencia en el procesamiento cognitivo de acuerdo a cada etapa (de Lira, Ortiz, Campanha, Bertolucci \& Minett, 2011; Ahmed, Haigh, Jager \& Garrard, 2013).

Tales exploraciones, además, han logrado contribuciones importantes en la detección temprana de la demencia gracias a los fenómenos lingüísticos que se han aceptado más significativos hasta el momento (López-de-Ipiña, Alonso, Travieso, 
Solé-Casals, Egiraun, Faundez-Zanuy \& Lizardui, 2013; Meilán, Martínez-Sánchez, Carro, López, Millian-Morell \& Arana, 2014; Zhu, Novikova \& Rudzicz, 2018).

Se tiene por consenso que el nivel pragmático-discursivo es una de las áreas prometedoras para estudiar la DTA; su utilidad reside en que el discurso es una de las manifestaciones ulteriores de la habilidad lingüística de todo sujeto, en donde se articulan cada una de las unidades de la lengua. En este sentido, resulta particularmente interesante ahondar en los mecanismos de unión y asociación que entran en juego durante la composición discursiva, especialmente en aquellos implícitos en la estructuración que dotan de sentidos específicos al discurso.

La construcción de tales significados ejecutados durante la transmisión de información en el discurso construido por el paciente con demencia constituye el principal interés de este trabajo. En primer lugar, proponemos que las relaciones de significado o relaciones retóricas encargadas de articular las partes del discurso de un adulto mayor se ven afectadas por el deterioro cognitivo causado por la DTA. En segundo lugar, creemos que las relaciones discursivas utilizadas para conectar el discurso varían entre la población con demencia tipo Alzheimer y la cognitivamente sana. En tercer lugar, especulamos que, durante la interacción entre adulto mayor y cuidador, se despliegan relaciones de significado que fungen como recursos de apoyo para la transición de la conversación.

Nuestro objetivo se centra, pues, en obtener las relaciones retóricas más características en el discurso conversacional de la población con DTA y la población cognitivamente sana y con ello, sopesar el grado de afectación que sufren tales relaciones en pacientes con esta demencia. En un segundo término, perseguimos identificar las relaciones retóricas con las que los cuidadores se sirven para optimizar y continuar con la conversación.

La utilidad de este trabajo se basa en el planteamiento de un método de análisis no invasivo y de bajo costo que examina la estructuración coherente y la conexión del discurso conversacional mediante los nexos semánticos denominados relaciones retóricas (Mann, Matthiessen \& Thompson, 1992; Mann \& Thompson, 1988). El estudio de la coherencia discursiva puede llegar a ser un adecuado y potencial auxiliar para el monitoreo y seguimiento de la Enfermedad de Alzheimer, a partir de lo cual se podrían mejorar y adecuar las estrategias comunicativas entre pacientes y familiares o cuidadores.

Los lineamientos de este trabajo se establecen a continuación. Para comenzar, hacemos un recuento de los principales trabajos enfocados a dilucidar fenómenos pragmático- discursivos para el perfilamiento lingüístico de la DTA. Posteriormente, a partir de los principios de coherencia, explicamos el concepto de relación retórica y su caracterización desde la perspectiva de la RST. Seguidamente, presentamos la metodología, la cual parte del proceso de transcripción de las conversaciones, y 
continúa con el análisis de la RST, las pruebas estadísticas de correlación y significación estadística y el recuento de frecuencias emitidas por los cuidadores. Los resultados extraídos de esas pruebas y su valoración se muestran en las últimas secciones de Resultados y Conclusiones, respectivamente.

\section{Antecedentes}

Si partimos desde los niveles de la lengua, quizás el nivel más estudiado para la Demencia tipo Alzheimer sea el léxico-semántico, nivel cuyo detrimento empieza desde las primeras etapas con el empobrecimiento de vocabulario, su fluidez y anomalías en el lexicón (Silagi, Bertolucci \& Ortiz, 2015; Kavé \& Goral, 2018). El escueto desempeño léxico se halla en el deterioro de la memoria semántica, la incapacidad para generar estructuras conceptuales prelingüísticas y la ruptura entre el concepto semántico y el referente (Ralph, Watson, Powis, Patterson \& Hodges, 2001; Grasso, Díaz \& Peraita, 2011; Szatloczki, Hoffmann, Vincze, Kálmán \& Pakaski, 2015).

Aunque existe un consenso en que el nivel fonético, es decir, la realización de los sonidos propios del sistema, suele preservarse en las primeras etapas de la enfermedad (Kempler, 1995), algunos trabajos hacen hincapié en que las parafasias fonéticas, la disartria y los alargamientos, aparecen desde los comienzos de la demencia (Sajjadi, Patterson, Tomek \& Nestor, 2012; Cera, Ortiz, Bertolucci \& Minett, 2018). La misma discusión se reanuda para el ámbito sintáctico pues, aunque el hablante es capaz de producir construcciones oracionales correctas, padece ciertas dificultades a medida que aumenta la complejidad de la oración (Boschi, Catricala, Consonni, Chesi, Moro \& Cappa, 2017; Mueller, Hermann, Mecollari \& Turkstra, 2018).

De igual modo que el nivel léxico-semántico, la esfera pragmática y discursiva se ha posicionado como uno de los niveles que más se atrofian conforme avanza la demencia (Garayzábal-Heinze, 2009). Esta es, pues, un área que devela valiosa información para su evaluación. Entre los primeros intereses se buscó conocer qué fenómenos hacían el discurso de un paciente más o menos coherente. Con el estudio de Nicholas, Obler, Albert y Helm-Estabrooks (1985), se comprobó que los déficits cohesivos que provocaban el discurso vacío en los pacientes con demencia tipo Alzheimer se centraban en pronombres sin antecedentes (deixis personal), parafasias semánticas, repeticiones y otros términos deícticos. Estos hallazgos fueron consistentes con los trabajos de Ripich y Terell (1988) y Ripich, Carpenter y Ziol (2000); en este último se remarca la disminución de elipsis y conjunciones (Hernández-Domínguez, et. al, 2018) en sujetos con DTA. Los déficits se agudizan en la etapa moderada de la demencia, pues al inicio los pacientes logran un relativo éxito manteniendo la conversación, es decir, conformando la representación mentalmente coherente de sus conversaciones (Orange, Lubinski \& Higginbotham, 1996). 
Al lado de la cohesión, las macroestructuras, que conciernen a la unificación temática del discurso, es decir, a la coherencia global, parecen encontrarse aún más atrofiadas (Laine, Laakso, Vuorinen \& Rinne, 1998). Las rupturas de turno se deben a la elisión de información que conecta al interlocutor con el tema en cuestión (Ripich \& Terell, 1988). Trabajos más recientes han concluido de igual manera que la coherencia global resulta ser una esfera particularmente conflictiva para la población con DTA (Dijkstra, Bourgeois, Allen \& Burgio, 2004; Toledo, et. al., 2018). Aunque más escasos, otros estudios también se han dedicado a examinar la coherencia local, es decir, la vinculación lógica entre estructuras del discurso (Lai, 2014; Brandão, Lima, Parente \& Peña-Casanova, 2017).

No solo se han examinado la cohesión y la coherencia en el discurso, sino las habilidades pragmáticas durante su ejecución. Van Lancker Sidtis, Choi, Alken y Sidtis (2015) examinan y descubren que la población con demencia, a diferencia de pacientes con Parkinson, recurría a las fórmulas conversacionales de manera atípicamente frecuente. Pérez Mantero (2015) analiza las transgresiones a las máximas conversacionales y las interacciones ocurridas en la conversación entre un paciente con Alzheimer y su cuidador (Pérez Mantero, 2014). A medida que progresa la demencia, encuentra que hay un incremento significativo de violaciones a las máximas; repara en que la inadecuación de las preguntas va en aumento a medida que la DTA avanza, lo que provoca pérdida de la coherencia global y devela la pérdida de la empatía del enfermo hacia su interlocutor.

Hays, Niven, Godfrey y Linscott (2010) evalúan los tipos de implicaturas que un paciente puede identificar; ya anteriormente Chapman, Highley y Thompson (1998) habían concluido que los hablantes con Alzheimer presentaban serias dificultades para formular inferencias, lo cual denotaba problemas para la extracción de contenido textual y la vinculación de este con el conocimiento del mundo del sujeto.

En contraparte a la pérdida de habilidades comunicativas, surgen trabajos que se enfocan en las aptitudes preservadas por esta población. Hamilton (1996) sostiene que a través de los recursos pragmáticos como las fórmulas de cortesía, cuidadores y familiares construyen una identidad de paciente al enfermo, lo cual limita su disposición a interactuar con ellos; Guendouzi y Müller (2006) concuerdan con tal observación, y afirman que al paciente con demencia se le predisponen roles de sujetos inactivos e inhábiles. Varela Suárez (2018) defiende que las preguntas propias de los pares adyacentes se mantienen incluso en las últimas etapas y como una estrategia pragmática consciente y dinámica de parte del paciente.

Por lo que respecta a los elementos prosódicos y suprasegmentales, estos se han descubierto y aceptado como una opción sumamente significativa al momento de descartar a sujetos con DTA. El abanico de estudios ha sido extenso, desde hesitaciones o pausas llenas o vacías (Davis \& Maclagan, 2009; Gayraud, Lee \& 
Barkat- Defradas, 2011; Pistono, Jucla, Barbeau, Saint-Aubert, Lemesle, Calvet \& Pariente, 2016); longitud de periodo de voz y tempo del discurso (Toth et al., 2018; Meilán et al., 2014; Hoffmann, Nemeth, Dye, Pákáski, Iriny \& Kálmán, 2010) y los problemas de entonación, como indicadores de intención y emociones (Taler, Baum, Chertkow \& Saumier, 2008; Tosto, Gasparini, Lenzi \& Bruno 2011; Meilán, MartínezSánchez, Carro, Sánchez \& Pérez, 2012).

En los últimos años, varios trabajos se han dedicado a explorar cómo se entrama la conexión discursiva de un hablante con DTA. Esto ha dado lugar al estudio del enfoque denominado como conexión del discurso (connected speech) (Sajjadi et al., 2012; Ahmed et al., 2013; Rentoumi, Raoufian, Ahmed, de Jager \& Garrard, 2014; Boschi et al., 2017; Mueller et al., 2018; Toth et al., 2018; Rochon, Leonard \& Goral, 2018; Slegers, Filiou, Montembeault \& Brambati, 2018). La mayoría de sus propuestas se basa en la integración de fenómenos lingüísticos de diversos niveles (la extracción léxica, tipos de unidades, contenido semántico, fenómenos suprasegmentales como entonación, etc.) con el fin de sopesar su eficacia conjunta en la identificación de la demencia.

Como se aprecia, no hay registro de que desde la conectividad del discurso o el análisis discursivo o conversacional se hayan considerado y analizado las relaciones discursivas o retóricas durante estas décadas de investigación. Actualmente, apenas un par de estudios se han enfocado en este tipo de demencia. Abdalla, Rudzicz y Hirst (2017) realizan un análisis automático que busca identificar patrones en las relaciones retóricas de población que sufría DTA y adultos cognitivamente sanos. Además de que la segmentación y el etiquetado automático tuvieron una evaluación exitosa, los autores descubren que la población con demencia producía una cantidad de relaciones cuantitativamente inferior; para ambas poblaciones, las relaciones de Elaboración, Unión y Atribución fueron de las más formuladas.

En poblaciones no angloparlantes, Paulino y Sierra (2017) y Paulino, Sierra, Hernández-Domínguez, da Cunha y Bel-Enguix (2018) realizaron estudios incipientes con la RST en un corpus de conversaciones de adultos mayores diagnosticados con demencia tipo Alzheimer y adultos cognitivamente sanos. Entre los resultados preliminares, resaltaron que, al igual que los datos de Abdalla et al. (2017), la relación de Elaboración era de las más utilizadas por los pacientes enfermos; junto con esta, la Concesión, la Causa voluntaria e involuntaria, la Solución y el Resultado involuntario resultaban escasamente formuladas por un adulto con Alzheimer.

\section{Marco teórico}

\subsection{Coherencia discursiva y relaciones retóricas}

La conectividad del discurso no depende únicamente de sus recursos cohesivos sino de la representación mental del texto, de los nexos, según las diversas unidades, 
que el hablante concibe del texto (Sanders \& Maat, 2006). Visto así, la conectividad discursiva, característica denotativa de la coherencia, es un proceso que se encuentra interconectado con otros procesos cognitivos en los que podemos incluir los procedimientos semánticos, funciones de ejecución y la memoria de trabajo (Mueller et al., 2018).

De una manera más puntual, la coherencia, desde la perspectiva de Valles González (2006: 182), se refiere a:

"la interpretación de un texto por un interlocutor, de acuerdo con la visión consistente del mundo, o mejor dicho, de acuerdo con un campo semántico compartido, que permite identificar e interpretar los nexos o vínculos entre las oraciones o proposiciones que conforman un determinado texto."

La coherencia, en cuanto a su alcance de aplicación, se escinde en coherencia global, que es el mantenimiento apropiado del tópico en el discurso, y la coherencia local, que se refiere a la interconexión entre cada proposición del discurso con respecto al tema general (Dijkstra et al., 2004; Lai, 2014; Brandão et al., 2017). Estas características también fueron señaladas por Van Dijk (1998). Desde otros matices en su conceptualización, se ha recalcado que, para la realización de la coherencia, es necesaria la explicación del contexto social en donde se lleve a cabo (Martin, 2001; Valles, 2009; Brandão et al., 2013). En general, en la teoría se tiene por acuerdo implícito el hecho de que tanto la cohesión como la coherencia son interdependientes. Sin embargo, la coherencia no depende del lenguaje en sí, del léxico o estructuras sintácticas, sino en la continuidad de hechos y eventos, de procesos discursivos de niveles superiores (Pietrosemoli, 1996).

Un proceso importante para entender el engranaje de la coherencia son las relaciones retóricas, pues la interpretación de segmentos relacionados requiere de más información que la suma de los segmentos en aislado (Sanders \& Maat, 2006; Lascarides \& Asher, 2008). Las relaciones retóricas son definidas como los enlaces de índole semántico, pragmático y funcional que se encargan de enlazar partes constitutivas del texto (Taboada \& Mann, 2006; Renkema, 2008; Das \& Taboada, 2014). Se trata de las relaciones subyacentes entre las proposiciones de un texto (Taboada, 2004); estas se encargan de ir conectando cada pieza funcional dentro del discurso, tal como lo recalca Garrido Medina (2007: 312):

"es necesario que los dos acontecimientos puedan formar parte de uno complejo. Esta es la clave de la conexión: se produce la relación en cuestión porque hay una manera de encajar los dos acontecimientos con las pistas lingüísticamente explícitas." 
La terminología de las relaciones y sus principios de clasificación se han denominado por otros autores como predicados retóricos (Grimes, 1975), relaciones de coherencia (Sanders \& Maat, 2006; Spooren \& Sanders, 2008; Kehler, Kertz, Rohde \& Elman, 2008) o relaciones retóricas (Mann \& Thompson, 1988). Junto con la terminología, también cambian los criterios de clasificación. Así, el catálogo de relaciones retóricas ha sido bosquejado por varios autores considerando clasificaciones que varían considerablemente en cuanto al número. Tales propuestas se basan en propiedades que comparten en mayor o menor grado (de contenido, interpersonales, entre segmentos del texto, de divergencia, de causa, o consecuencia, por ejemplo), aunque también integran consideraciones nuevas, como actos de habla (imperativa, declarativa, etc.) (Lascarides \& Asher, 2008), heterocorrecciones e implicaciones de índole pragmática, como bromas (Stent \& Allen, 2000).

\subsection{Rhetorical Structure Theory}

El método de análisis que aplicamos en este trabajo es la Rhetorical Structure Theory (en español, Teoría de la Estructura Retórica), conocida mayormente como RST. Como sus autores señalan, la RST trata de una "aproximación lingüística descriptiva para un conjunto de fenómenos en la organización del discurso”. Específicamente, Taboada y Mann (2006: 425) señalan que:

"explains coherence by postulating a bierarchical, connected structure of texts, in which every part of a text has a role, a function to play with respect to other parts of text."

Debido a la modalidad de nuestra muestra, optamos aquí por la propuesta de Taboada (2004), la cual es reformulada para discursos de carácter oral. El engranaje de la teoría se basa en ciertos criterios teóricos: ‘unidades semánticas de diálogo' (SDU) para textos orales, los 'esquemas' o patrones de organización, los spans o grupos mínimos funcionales de estructuras y las 'relaciones retóricas'.

\subsubsection{Unidades semánticas de diálogo (SDU), esquemas y spans}

La teoría tradicional de la RST denomina 'unidades mínimas de discurso' a las unidades fundamentales del texto. Suelen identificarse, grosso modo, como las cláusulas de las oraciones (Das \&Taboada, 2014) aunque, en realidad, no son estos los únicos criterios que se han tomado para su identificación, tal como lo recalcan algunos autores:

"While some take the elementary units to be clauses [...] in others take them to be prosodic units [...] turns of talk [...] sentences [...] intentionally defined discourse segments [...] or the "contextually indexed representation of information conveyed by a semiotic gesture, asserting a single state of affairs or partial state of affairs in a discourse world. (Polanyi, 1996: 5)" (citado en Carlson, Marcu \& Okurowsky, 2003: 3). 
Esto quiere decir que los criterios de segmentación son perfilados por la modalidad discursiva. Para los textos escritos, los criterios estructurales son particularmente útiles. Casi todos los autores concuerdan como criterio válido localizar cláusulas oracionales (con excepción de aquellas que son sujetos u objetos de un verbo principal en oraciones subordinadas), verbos conjugados y marcadores del discurso. Sin embargo, las implicaciones de cláusula u oracionales son superadas al momento de segmentar discursos hablados. Ante esto, se han propuesto estructuras dialogales como pares adyacentes y fenómenos prosódicos (Stent \& Allen, 2000).

Como ya se dijo, Taboada denomina a las unidades mínimas fundamentales del discurso conversacional, unidades semánticas de diálogo (SDU). Tales unidades se rigen por los criterios clásicos de oraciones, cláusulas (de circunstancia, tiempo, condicionales, etc.) y verbos conjugados, pero también por fenómenos prosódicos y de estructura conversacional. La autora equipara tales unidades con las information units ${ }^{1}$ de Halliday (1967), o las intonation units ${ }^{2}$ de Chafe (1994).

Estas unidades, al momento de relacionarse a través de una estructura retórica, se disponen de una forma particular y acorde a su jerarquía. A la organización entre las unidades se le denomina 'esquema'; básicamente representan todas las estructuras posibles que pueden darse entre unidades (Taboada, 2004). Cuando tales estructuras se dan, se ha formado un pequeño grupo de relaciones conectadas. Los spans representan estos conjuntos de unidades ya organizadas.

\subsubsection{Relaciones retóricas}

Taboada se basa en la clasificación actualizada de relaciones propuesta por Mann y Thompson (1988), la cual consta de 32 relaciones ${ }^{3}$ (véase Tabla 1). Las pautas para su clasificación están regidas por dos propiedades. La primera de ellas, y más general, tiene que ver con el nivel jerárquico de unidades enlazadas: si la asociación entre unidades semánticas se da entre una, cuya importancia es mayor, y la otra, que a su vez redondea o complementa a la primera, entonces la relación en cuestión es de índole nuclear $(\mathrm{N}+\mathrm{S})$. $\mathrm{Si}$, por el contrario, los segmentos poseen un nivel jerárquico equiparable (y de estructura similar, en la mayoría de ocasiones) entre ellos, luego, la relación que los enlaza es de carácter multinuclear $(\mathrm{N}+\mathrm{N})$. 
Tabla 1. Clasificación actualizada de las relaciones retóricas propuestas por Mann y Thompson (1988).

\begin{tabular}{|l|l|l|l|l|}
\hline \multicolumn{2}{|c|}{ Nucleares } & \multirow{2}{*}{ Multinucleares } \\
\hline \multirow{2}{|c|}{ De presentación } & \multicolumn{2}{c|}{ De contenido } & \\
Antítesis & Justificación & Alternativa & Medio & Contraste \\
Capacitación & Motivación & Causa invol. & No condicional & Lista \\
Concesión & Preparación & Circunstancia & Resultado invol. & Reformulación \\
Evidencia & Reformulación & Condición & Propósito & multinuclear \\
Fondo & Resumen & Elaboración & Solución & Unión \\
& & Evaluación & Unless & Conjunción \\
& & Interpretación & & Disyunción \\
\hline
\end{tabular}

El siguiente punto corresponde a los propósitos generales para con el discurso; si el propósito de las relaciones va orientado a persuadir de algún modo al interlocutor (función pragmática), serán relaciones de presentación (o presentacionales). Si la función de las relaciones, por el contrario, se enfoca únicamente en dar cuenta del funcionamiento interno del discurso (carácter semántico), entonces se tratarán de relaciones de contenido (también denominadas relaciones de sujeto o de asunto tratado). Ejemplos de las relaciones Concesión (ejemplo 1), Reformulación (ejemplo 2), Circunstancia (ejemplo 3), Causa voluntaria (ejemplo 4) y Lista (ejemplo 5) son expuestos a continuación; se señala en cada caso los tipos de unidad (nuclear o satélite).

(1) Sra. Del Carpio: <claro> Entonces [pron=tonces], ah, ese departamentito, - eh, pedí yo a mi hijo para - por medio de escritura, [N] | con todo yo les agregué un cuarto para llegar cuando yo quiera. [S]

(2) Sra. Restrepo: \#2 Sí estoy tranquila, sí sí estoy tranquila. \# [N] | Sí estoy bien, para que tam $\sim$ [risas]. [S]

(3) Sra. Luna: Me gustaba cuando - íbamos a hacer algunas novelas, [S] | me gustaba mucho venir con mi esposo. [N]

(4) Sr. Murrieta: Yo, en primer lugar soy feliz [N] | porque los tengo a mis dos hijos $<y a>$. [S]

(5) Sra. Restrepo: "No mamá, ya no voy a tomar, [N] | ya voy a componerme, $[\mathrm{N}]$ | ya me voy a hacer de cenar". [N]

\section{Marco metodológico}

\subsection{Muestra}

Nuestra muestra fue tomada del corpus Carolinas' Conversations en español, recopilado por los investigadores Hernández-Domínguez, Ratté, Davis y Pope (2016). El corpus cuenta con alrededor de treinta grabaciones de conversaciones entre cuidadores y adultos mayores hispanohablantes que sufren demencia tipo Alzheimer 
en alguna de sus fases, y adultos mayores en un estado cognitivamente sano. Estas grabaciones fueron realizadas con el consentimiento informado de los participantes y, en el caso de los pacientes con DTA, de sus guardianes legales, siguiendo un protocolo aprobado por el comité de ética en la investigación de la École de Technologie Supérieure de la Universidad de Quebec. Para toda la muestra, dos cuidadoras fueron las moderadoras de la conversación; ambas eran hispanohablantes y de sexo femenino.

Por cuestiones de delimitación y disponibilidad, las grabaciones utilizadas corresponden al año 2015. El intervalo de duración de los audios oscila entre los seis y cincuenta minutos. En total, tomamos siete pacientes con DTA en alguna de sus fases y seis adultos cognitivamente sanos. Todos los pacientes se encuentran en un rango de edad que oscila entre los ochenta a noventa años y son originarios de Ecuador. La Tabla 2 compila, además, los datos clínicos.

\subsection{Transcripción del corpus}

Seguimos las convenciones de transcripción sugeridas por el propio corpus de Carolinas' Conversations Collection. Se optó por conservar ese sistema de anotación debido a su alto grado de inteligibilidad. Los nombres reales de los pacientes fueron sustituidos por nombres de personajes literarios de un catálogo compilado previamente. La trascripción se hizo a nivel ortográfico y prosódico: pausas, pausas llenas, cambios de tono (agudo, grave), entonaciones, volumen, énfasis, etc.; además, se tuvieron en cuenta fenómenos quinésicos (movimiento de manos, gestos, posiciones) y proxémicos (cercanía entre el cuidador y el paciente) que incidieran de forma directa en la conversación. La estructura base para la transcripción fue el turno. Tomamos este como una unidad estructural de ordenamiento atendida y aceptada entre los interlocutores. Todo el proceso de etiquetado y la exportación de los archivos en formato de texto plano fue realizado con ayuda del programa Transcriber ${ }^{4}$.

\subsection{Identificación y segmentación de unidades}

La segmentación de unidades consistió en dos revisiones. Primero se identificaron núcleos verbales (verbos conjugados), cláusulas (con excepción de las que fueran atributo de sujeto y objeto del núcleo verbal en estructuras complejas) y nexos o marcadores discursivos. Algunos signos de puntuación (que intuitivamente se utilizaron en la conversación para marcar la delimitación de una idea terminada) de igual forma se tomaron en cuenta al momento de la segmentación: 
Tabla 2. Datos sociolingüísticos y diagnóstico clínico de la muestra.

\begin{tabular}{|c|c|c|c|c|c|c|c|}
\hline No. & Alias & Género & Edad & Escolaridad & $\begin{array}{l}\text { Lengua } \\
\text { materna }\end{array}$ & $\begin{array}{l}\text { Lugar de nacimiento } \\
\text { y de residencia actual }\end{array}$ & Datos clínicos \\
\hline 1 & $\begin{array}{l}\text { Joaquín } \\
\text { Murrieta. }\end{array}$ & Masculino & 81 & Licenciatura & Español & $\begin{array}{l}\text { Loja Ecuador- Quito, } \\
\text { Pichincha. }\end{array}$ & $\begin{array}{l}\text { Alzheimer moderado, } \\
\text { Diabetes, Hipertensión }\end{array}$ \\
\hline 2 & $\begin{array}{l}\text { Petra Cor- } \\
\text { tés }\end{array}$ & Femenino & 84 & $*$ & Español & $\begin{array}{l}\text { Guayaquil, Ecuador- } \\
\text { Quito, Pichincha }\end{array}$ & $\begin{array}{l}\text { Alzheimer avanzado, } \\
\text { Hipertensión }\end{array}$ \\
\hline 3 & $\begin{array}{l}\text { Susana San } \\
\text { Juan }\end{array}$ & Femenino & 82 & * & Español & $\begin{array}{l}\text { Quito, Fcuador- Quito } \\
\text { Pichincha }\end{array}$ & $\begin{array}{l}\text { Alzheimer moderado, } \\
\text { Diabetes, Hipertensión }\end{array}$ \\
\hline 4 & $\begin{array}{l}\text { Ángela Vi- } \\
\text { cario }\end{array}$ & Fernenino & 81 & $*$ & Español & $\begin{array}{l}\text { Tulcán, Ecuador- Quito, } \\
\text { Pichinchá }\end{array}$ & $\begin{array}{l}\text { Alzhleimer, Hiperten- } \\
\text { siónı }\end{array}$ \\
\hline 5 & Luisa Mora & Femenino & 90 & * & Español & $\begin{array}{l}\text { Quito, Ecuador- Quito, } \\
\text { Pichincha }\end{array}$ & $\begin{array}{l}\text { Alzhcimer, Diabetes, } \\
\text { Hipertensión }\end{array}$ \\
\hline 6 & $\begin{array}{l}\text { Ana Zama- } \\
\text { cona }\end{array}$ & Femenino & 84 & $*$ & Español & $\begin{array}{l}\text { Quito, Ecuador- Quito, } \\
\text { Pichincha }\end{array}$ & $\begin{array}{l}\text { Alzheimer moderado, } \\
\text { Depresión, Hipertensión }\end{array}$ \\
\hline 7 & $\begin{array}{l}\text { José 13uen- } \\
\text { día }\end{array}$ & Masculino & 90 & * & Español & $\begin{array}{l}\text { Ibarra, Ecuador- Quito, } \\
\text { Pichincha }\end{array}$ & Alzheimer leve \\
\hline 8 & $\begin{array}{l}\text { Rosenda } \\
\text { Zubarán }\end{array}$ & Femenino & 91 & $\begin{array}{l}\text { Primaria, } 2 \\
\text { años de of cio }\end{array}$ & Español & $\begin{array}{l}\text { Otavalo, Ecuador- Qui- } \\
\text { to, Pichincha }\end{array}$ & Hipotiroidismo \\
\hline 9 & $\begin{array}{l}\text { Fernanda } \\
\text { DelCarpio }\end{array}$ & Femenino & 80 & $\begin{array}{l}\text { Secundaria } \\
\text { técnica, of cio } \\
\text { de contadora }\end{array}$ & Español & $\begin{array}{l}\text { Quito, Ecuador- Quito, } \\
\text { Pichincha }\end{array}$ & Diabetes, Hipertensión \\
\hline 10 & $\begin{array}{l}\text { Ronata Re- } \\
\text { medios }\end{array}$ & Femenino & 88 & Primaria & Español & $\begin{array}{l}\text { Chimborazo, Ecuador- } \\
\text { Quito, Pichincha. }\end{array}$ & $\begin{array}{l}\text { Hipoliroidismo, } \\
\text { Diabetes }\end{array}$ \\
\hline 11 & Eva Luna & Femenino & 81 & $*$ & Español & $\begin{array}{l}\text { Quito, Ecuador, Quito, } \\
\text { Pichinchia }\end{array}$ & $*$ \\
\hline 12 & $\begin{array}{l}\text { Isabel } \\
\text { Allende }\end{array}$ & Femenino & 85 & $\begin{array}{l}\text { Primaria, } \\
\text { años }\end{array}$ & Español & $\begin{array}{l}\text { Quito, Ecuador- Quito, } \\
\text { Pichincha }\end{array}$ & Diabetes, Hipertcnsión \\
\hline 13 & $\begin{array}{l}\text { Laura Res- } \\
\text { trepo }\end{array}$ & Femenino & 79 & $\begin{array}{l}\text { Primaria, } \\
\text { años }\end{array}$ & Español & $\begin{array}{l}\text { Quito, Ecuador- Quito, } \\
\text { Pichincha }\end{array}$ & Hipertensión \\
\hline
\end{tabular}

(6) Sra. Zubarán: Mi vida de niña feliz porque mi papá, mi mamá me querían harto, más que a ninguna de mis hijas. <la mimada $>$ [N] | Éramos nueve, nueve hermanos éramos, [S] | pero yo fui la más mimada. [S]

En el ejemplo 6, la intervención de la paciente Zubarán tiene tres unidades. Aunque la primera SDU podría segmentarse en más de una debido al fenómeno de elipsis verbal, nuestro criterio de partida para ambas poblaciones fue identificar la integridad de una unidad tanto como sea posible. Con este adulto es evidente el uso de la elipsis como estrategia cohesiva; sin embargo, el mismo fenómeno sería ambiguo de identificar y diferenciar en un paciente con demencia. Preferimos entonces conservar en este ejemplo la primera unidad como una sola, y únicamente segmentar en casos evidentemente necesarios, puesto que, al fin y al cabo, la formulación completa de una SDU da cuenta de la correcta estructura de emisiones de un paciente.

No todas las estructuras eran claras en cuanto a los criterios estructurales. En ocasiones, los límites de un segmento resultaban confusos; cuando esto era así, considerábamos cambios tonales, entonaciones, cambios en la frecuencia de emisión y la duración de pausas. De igual manera, Taboada (2004) localiza ciertos fenómenos en la estructura de la conversación que tomamos en cuenta para la segmentación: 
a) Enunciados de un solo span. Si un turno estaba constituido por una SDU, tales unidades no se consideraban para el etiquetado de relaciones:

(7) Sra. Daconte: Hasta ahora.

Sra. Zubarán: Hasta ahora ha que ya no viven mis papás. [N]

b) Monólogos. Unidades emitidas por el hablante en turno que no poseen función ilocutiva sino están enunciadas para sí mismo o, en su defecto, pretenden no perder la toma de turno. En el ejemplo 8, en la respuesta del paciente Buendía, la primera SDU está interrumpida por una intervención que el hablante emite para sí (“¿Cómo digo?”), posteriormente se reanuda y completa la unidad. En estos casos tal unidad se incluía dentro del segmento principal, puesto que para nuestros propósitos, no era funcional en aislado.

(8) Sra. Balceta: Dele pues.

Sr. Buendía: Una, dos, tres [risas]. | No, en verdad, nada más [pron=nomás] vivía en, ¿cómo digo? Fuera de la casa; $[\mathrm{N}]$ | venía - cada mes, $<y a>$ a dejarle el dinero a la señora y me iba. [S]

c) Relaciones paralelas. Se dan particularmente cuando se establecen pares adyacentes: pregunta-respuesta, petición-respuesta, etc. Optamos por separar esta clase de estructuras y únicamente tener en cuenta para el análisis a las unidades verdaderamente funcionales.

(9) Sra. Balceta: \#1 Y ahora yo [laughs]. |Y así me niega que no me quiere, pícaro este. [N]| No puede ser. [S]

d) Fragmentos del discurso específicos del género. En las conversaciones, las fórmulas de cortesía tales como saludos, introducciones prehechas o despedidas resultan indispensables para su correcto seguimiento. En algunos casos, las fórmulas constituían unidades semánticas, de tal forma que sí se contaban para el etiquetado.

A lo largo de la segmentación hemos identificado otros fenómenos que, aunque usuales en la conversación oral, se acentúan en las conversaciones entre pacientes enfermos e inciden en el proceso de división: frases a medias, falsos comienzos o balbuceos, repeticiones, intervalo de duración de pausas, circunloquios o enunciados interrumpidos. Ante estos casos, siempre consideramos el principio de integridad funcional o completud semántica (Kong, Linnik, Law \& Shum, 2017); en otras palabras, buscamos que una unidad fuera entendible para nosotros como anotadores, aunque no tuviera una estructura canónica (Lai, 2014). 


\subsection{Etiquetado de relaciones}

El proceso de asignar las relaciones retóricas se basa en lo que Garrido Medina denomina la 'lógica del discurso' y tiene que ver con la información que necesita una unidad de otra u otras para adquirir sentido (2007: 311):

"las relaciones entre oraciones que constituyen el discurso se infieren en el proceso de conexión [...] la característica constitutiva del discurso es que relaciona las oraciones que lo forman de una manera definida, de una determinada manera."

Identificar primeramente las SDU más generales y significativas de cada turno es fundamental para formar las posteriores jerarquías; generalmente, cada SDU jerárquicamente superior suele ser complementada o exigir información adicional que será proporcionada por SDU adyacentes a ella. En el análisis se hizo tanto la segmentación como el etiquetado de cuidadores y de pacientes.

\subsection{Construcción de árboles discursivos}

La asociación semántica de las SDU que conformaban spans fue la base para la construcción de diagramas más complejos y generales.

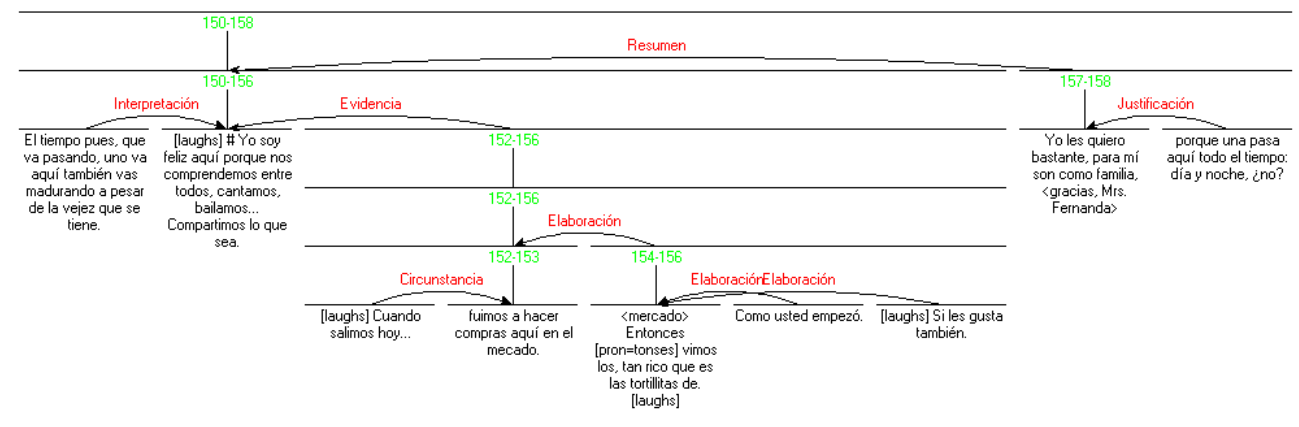

Figura 1. Mapa arbóreo del turno de un paciente sano.

La Figura 1 esquematiza un árbol discursivo que representa un turno completo. La relación más importante y general es de Resumen, una relación que implica una reformulación de lo que anteriormente se ha dicho. En el diagrama, la SDU 151 es la unidad más significativa, en donde recalca la felicidad de pertenecer a la comunidad de adultos mayores. El elemento anterior es una especie de reflexión, que puede representarse mediante la etiqueta de Evaluación (150). A manera de Evidencia, la hablante relata una anécdota sobre unas compras al mercado (153) y nos da antes el contexto temporal (152). De ella depende una relación de Elaboración sobre una comida (154), y de esta se desprenden los dos últimos satélites que simplemente puntualizan a quiénes les gusta el platillo $(155,156)$. Todo ese gran span tiene por motivación demostrar lo enunciado en la SDU 157, que resume lo dicho en la SDU 
151. Como extra, la hablante da nuevamente una Justificación breve (157) que remata el Resumen inmediatamente anterior.

\subsection{Análisis estadístico}

El procedimiento estadístico consistió en la normalización del corpus a través de valores relativos; tal procedimiento, además, nos permitió realizar una contabilización de producción de frecuencias para cada relación. Para conocer la asociación de la producción de una u otra relación al estado enfermo o sano del hablante, se realizó un análisis de correlación. Después de la evaluación, fue necesario obtener el grado mínimo de significación estadística de los resultados con respecto a nuestra muestra. Además de hacer el análisis de la RST para las poblaciones, se obtuvieron las SDU y las relaciones utilizadas por los cuidadores con el fin de comparar sus relaciones y tipos con respecto a los adultos mayores. Únicamente se requirió la contabilización de frecuencias y sus valores absolutos con cada relación.

\section{Resultados}

El Gráfico 1 muestra la frecuencia de relaciones entre ambas poblaciones. De entrada, sobresale una relación que predominó en el discurso de los sujetos sanos y los enfermos: la Elaboración. Tal relación es cuantitativamente superior al resto de relaciones, si bien la población sana la formuló en mayor medida (26,0\% sobre 23,4\%) (véase ejemplo 10). La segunda relación más formulada por ambas poblaciones fue la Reformulación (9,3\%, sanos y 8,6\%, con DTA) (véase ejemplo 11). A partir de estas dos relaciones, las frecuencias en la formulación de una y otra población discrepan, pues mientras las poblaciones sanas optan por la Evidencia $(6,8 \%)$ en su conversación (véase ejemplo 8), los que presentan demencia registraron la relación de Justificación $(7,6 \%)$ (véase ejemplo 12). En un cuarto puesto, la Concesión $(5,8)$ y el Fondo $(5,5 \%)$ fueron las más producidas por los pacientes sanos y con demencia, respectivamente.

(10) Sra. Allende: Teníamos dos almacenes de calzado [N] | y en uno trabajaba él [S] | y en otro trabajaba yo $<$ ahh $>$. [S]

(11) Sra. Allende: [...] porque yo [pron=entonación descendente] me retiro. [N] | Yo me voy a mi casa. [S]

(12) Sra. Buendía: Yo no sabía de, de la, del campamento que había donde se hizo la rut , [S] | salía cada mes acá a la casa, <ya> acá a Quito. [N]

La prueba estadística de correlación de Pearson se muestra en la Tabla 3. Sus resultados nos muestran cuál es la correlación positiva o negativa (inversa) que registra cada relación con respecto al estado sano o enfermo del paciente. La correlación positiva y cercana a 1 , da cuenta de la relación asociada a un paciente sano, cognitivamente hablando, mientras que la relación registrada con un valor cercano a 1, denota una correlación negativa que se asocia a la emisión de un paciente con DTA. 
Adicionalmente, integramos el valor de significación estadística (p-value) de cada correlación en nuestro corpus. Si una relación correlacionada positiva o negativamente tiene una significación estadística igual o menor a .05 , entonces podremos decir que la correlación es estadísticamente significativa.

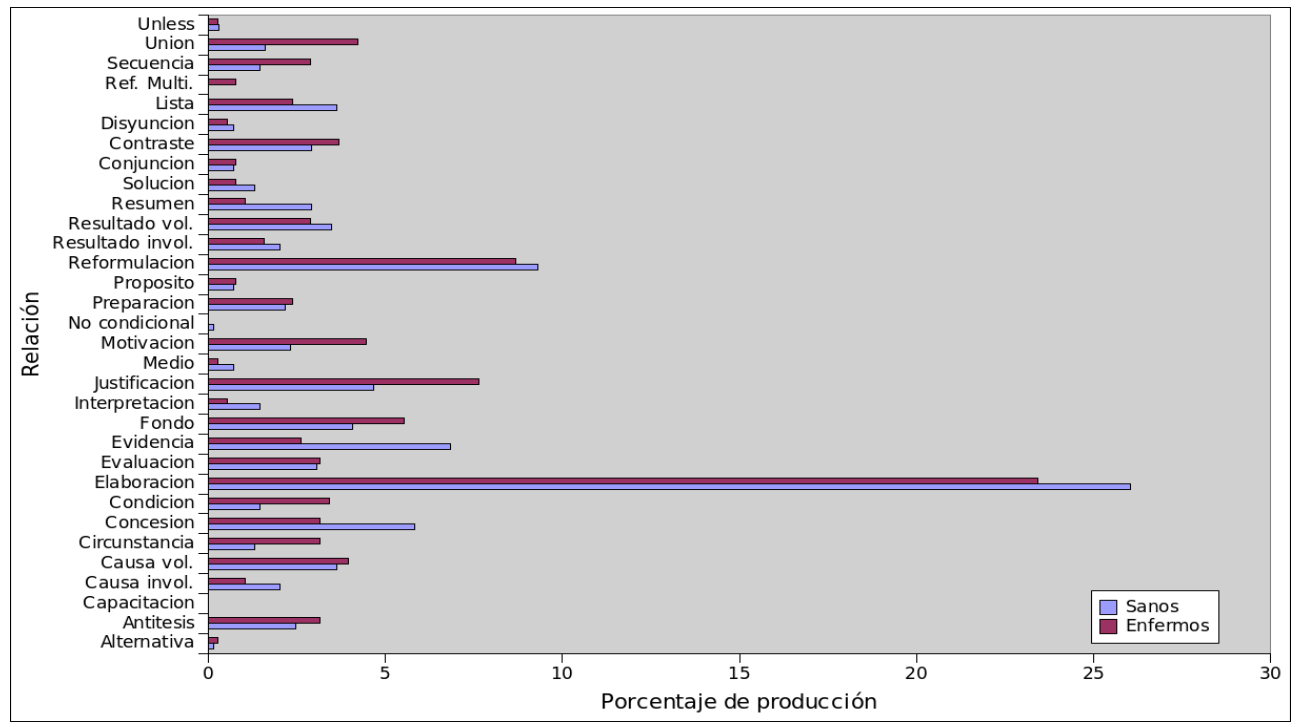

Gráfico 1. Producción de relaciones retóricas en pacientes con DTA y en adultos cognitivamente sanos.

Con el fin de confrontar los resultados encontrados en el trabajo de Abdalla et al. (2017), anexamos como variables, a la par de cada una de las relaciones retóricas, los fenómenos Total_multinucleares, Total_nucleares y Total. Esta inclusión también nos permite reiterar qué población es la que más relaciones emite y si tal proposición puede considerarse una constante en su discurso conversacional. 
Tabla 3. Correlación de variables y p-value.

\begin{tabular}{lrr|lrr}
\hline \multicolumn{1}{c}{ feature } & \multicolumn{1}{c}{ p_corr. } & \multicolumn{1}{c}{ p- value } & feature & p_corr. & p- value \\
\hline Concesion & 0.7982079443 & 0.001072393 & Resultado vol._\% & -0.0669895 & 0.827866 \\
Total_nucleares & 0.7129845439 & 0.006225043 & Resultado invol._\% & -0.0758963 & 0.8053501 \\
Total & 0.703015023 & 0.00735242 & Resumen_\% & -0.0922039 & 0.7644926 \\
Causa invol. & 0.6984995811 & 0.007911551 & Condicion_\% & -0.113332 & 0.7123944 \\
Solucion & 0.695328802 & 0.008323154 & Unless_\% & -0.1133435 & 0.7123662 \\
Resultado invol. & 0.642467548 & 0.017884118 & Preparacion_\% & -0.1279962 & 0.6768849 \\
Causa invol._\% & 0.6392188273 & 0.018662304 & Multinucleares_\% & -0.1443535 & 0.637992 \\
Interpretacion & 0.6382983723 & 0.018887308 & Conjuncion_\% & -0.1537952 & 0.6159173 \\
Lista & 0.6122608823 & 0.026129511 & Evaluacion_\% & -0.1580508 & 0.6060626 \\
Circunstancia & 0.6086053178 & 0.027289907 & Secuencia_\% & -0.1703338 & 0.5779661 \\
Solucion_\% & 0.6059853239 & 0.028144606 & Contraste_\% & -0.1946874 & 0.5238833 \\
Resumen & 0.602300651 & 0.029379715 & Justif cacion_\% & -0.2023004 & 0.5074483 \\
Fondo & 0.6009029028 & 0.029858493 & Union_\% & -0.2355576 & 0.4384952 \\
Resultado vol. & 0.5895587784 & 0.033958475 & Motivacion_\% & -0.2451717 & 0.4194682 \\
Elaboracion & 0.5744757255 & 0.04002691 & Alternativa & -0.2672612 & 0.3773739 \\
Evidencia & 0.5168588755 & 0.070508975 & Alternativa_\% & -0.2672612 & 0.3773739 \\
Total_multinuc. & 0.514891253 & 0.07177376 & Reformulacion_\% & -0.3215253 & 0.2840601 \\
Circunstancia_\% & 0.5032123461 & 0.079609036 & & & \\
Contraste & 0.4939129873 & 0.086258235 & & & \\
Interpretacion_\% & 0.4897416303 & 0.089361693 & & & \\
Fondo_\% & 0.4833061545 & 0.094298944 & & & \\
Causa vol. & 0.467001914 & 0.10763569 & & & \\
Preparacion & 0.4303766834 & 0.142105695 & & & \\
Conjuncion & 0.4303766834 & 0.142105695 & & & \\
\hline
\end{tabular}

En la Tabla 3 se visualizan tres columnas; estas señalan las variables (primera columna), los valores de correlación (segunda columna) y el nivel de significación estadística (tercera columna). El primer bloque de relaciones que va de Concesión a Evidencia posee un 95\% de significación estadística; estas relaciones son Concesión, Causa involuntaria, Solución, Resultado involuntario, Interpretación, Lista, Circunstancia, Resumen, Fondo, Elaboración y Evidencia. También aparecen las variables Total_multinucleares y Total, lo cual nos permite aseverar que, mientras más sano se encuentre un adulto mayor, mayor cantidad de relaciones formulará. Este grupo de relaciones, entonces, está correlacionado con el discurso de un paciente mentalmente sano.

El siguiente grupo de relaciones, que va de la variable Total_multinucleares a la relación de Fondo, posee un $90 \%$ de significación estadística y también está correlacionada con el estado mentalmente sano de un paciente. El último bloque de la tabla, que va de Justificación a Reformulación, tiene una correlación negativa, lo que implica que los pacientes con demencia formulan en su discurso tales relaciones. Para este bloque, sin embargo, el valor de significación no fue menor o igual a 0.05.

Junto con el recuento de frecuencias en las conversaciones de pacientes, nos pareció relevante conocer cuántas relaciones y cuáles son formuladas por sus respectivos cuidadores; la tarea es meramente exploratoria y nos motivamos por el perfil constante que los cuidadores mantienen en tanto moderadores. Únicamente fue 
necesario realizar la contabilización de la frecuencia de las relaciones y observar cuáles fueron formuladas en mayor medida. Los resultados se muestran en el Gráfico 2.

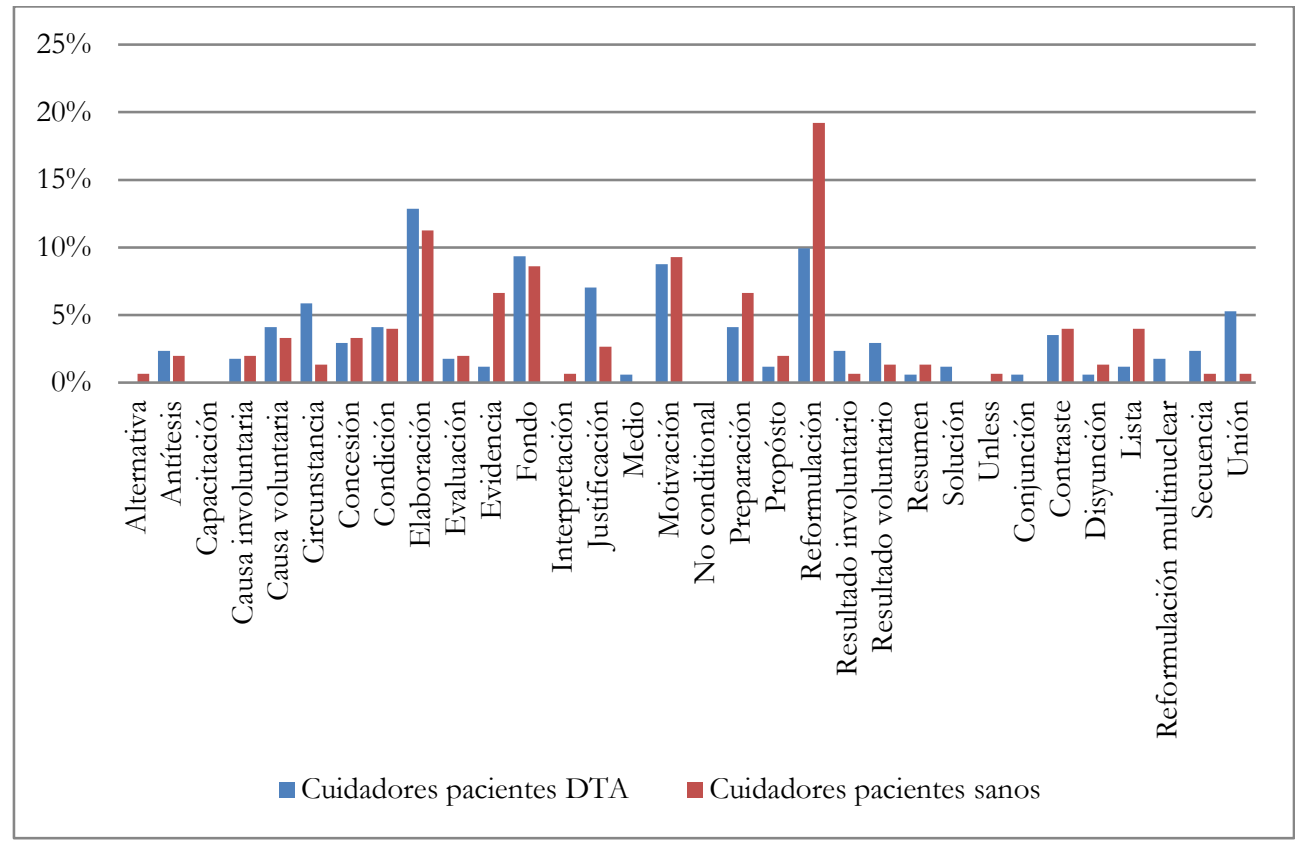

Gráfico 2. Producción de relaciones retóricas de cuidadores en ambas poblaciones.

Como se ve, el recuento muestra resultados dispares: en ciertas relaciones, los entrevistadores que conversan con pacientes con DTA superan en frecuencia a la población sana (Causa voluntaria, Circunstancia, Elaboración, Fondo, Unión, por ejemplo). En otras más, la producción de relaciones de los cuidadores en conversaciones con interlocutores sanos es la que rebasa sus intercambios con respecto a pacientes con demencia (Evidencia, Motivación, Preparación y Reformulación, Lista). Entre los valores de la gráfica hay, sin embargo, una constante: en ambas poblaciones, los cuidadores producen mayor cantidad de relaciones de Elaboración, Fondo, Motivación y Reformulación.

Nuevamente, la Elaboración, junto con la Reformulación, son las relaciones con mayor frecuencia de uso. El ejemplo 13 ilustra el empleo de ambas en la tarea de formulación de preguntas para el paciente:

(13) Sra. Daconte: A ver, ¿cómo, cómo ha sido su vida? [N] | Si ha sido buena, agradable, [S] | si usted volvería a hacer lo mismo que ha hecho, [S] | no lo volvería a hacer, [S] | a ver, ¿cómo fue su vida? [S]

La relación principal de la cuidadora Daconte, que indaga por la vida del paciente (“¿cómo ha sido su vida?'), es desarrollada en los siguientes segmentos de su intervención ('si usted volvería a hacer lo mismo'; 'si no lo volvería a hacer'), seguramente con el objeto de asegurar que el paciente comprenda la implicación de 
esa pregunta. Al final del turno, vemos que la última unidad vendría a ser, básicamente, la reiteración del núcleo, razón por la cual se interpreta como una Reformulación, relación que es frecuente encontrar en las preguntas del cuidador (ejemplos 14, 15 y 16):

(14) Sra. Balceta: ¿Qué tal pasa aquí en esta casa hogar? - [N] | ¿Le gusta vivir aquí? [S]

(15) Sra. Balceta: O sea que con, y cuando se va usted de aquí, [S] | ¿con quién se va?, [N] | ¿en qué casa pasa: donde Sra. Eduviges o donde Sra. Daminana? [S]

(16) Sra. Daconte: Su hijo. ¿Y usted dónde se encuentra ahorita, dónde está? [N] | ¿Sabe dónde está? [background conversations] [S]

En la Figura 2, observamos de igual manera relaciones recurrentes en los cuidadores; destacan los spans de Motivación y Fondo para asentar con seguridad la pregunta nuclear de las unidades 407- 408. Así, la Circunstancia y el Fondo denotan el contexto, el conocimiento compartido entre ambos, y la Motivación se recalca como una petición. Los usos de esta serie de relaciones fueron sumamente constantes a lo largo del análisis entre cuidadores.

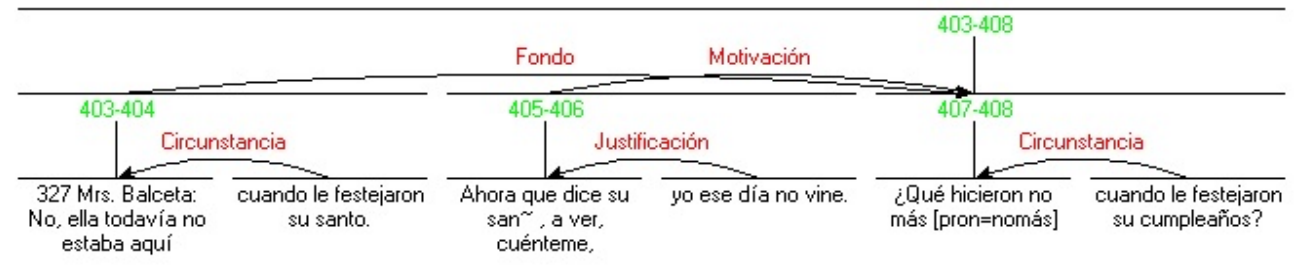

Figura 2. Diversos tipos de relaciones formuladas en el discurso del cuidador.

\section{Discusión}

Nuestros resultados dieron respuesta a varios de nuestros planteamientos iniciales. De entrada, con respecto al detrimento que esta clase de demencia produce en la formulación del discurso y, más específicamente, en las relaciones de significado subyacentes, pudimos constatar que un adulto mayor en estado cognitivo sano es capaz de formular en su discurso conversacional más relaciones nucleares y multinucleares, y de más tipos (Abdalla et al., 2017). Lo anterior quiere decir que un hablante cognitivamente sano puede formular más fácilmente estructuras semánticamente adecuadas (Sajjadi et al., 2012) y, por ende, la estructuración de su discurso quedará regida por las reglas cognitivas lingüísticas requeridas (Mueller et al., 2018).

La preservación de la coherencia discursiva resulta significativa porque conlleva directamente a otras habilidades lingüísticas preservadas por los hablantes sanos, por ejemplo, la adecuación en el uso de los mecanismos de cohesión: la identificación de 
unidades estructurales con contenido semántico (t-units, semantic units, etc.), ya destacados por Mueller et al. (2018) como un fenómeno clave en la identificación de la demencia, la capacidad para utilizar elementos cohesivos, como el uso de pronombres y otros elementos deícticos, conjunciones y nexos, en general (Dijkstra et al., 2004). Por tanto, la coherencia es una propiedad que permite hilar o vincular otras habilidades lingüísticas y cognitivas.

En segunda instancia, formulamos que las relaciones discursivas utilizadas para conectar el discurso varían entre la población con demencia tipo Alzheimer y la cognitivamente sana. Por lo que respecta a la primera población, obtuvimos indicios de que los adultos mayores con esta demencia formulan en su discurso más relaciones de Justificación, Unión, Alternativa y Reformulación; con todo, el p-value no proporcionó la significación estadística necesaria para comprobar tal afirmación.

Preliminarmente y ateniéndonos a los hallazgos de futuros avances, podemos decir que algunas de estas relaciones guardan congruencia con las habilidades preservadas por los sujetos con DAT. Por ejemplo, de las relaciones de índole multinuclear incluidas en la clasificación de Mann y Thompson, la Unión, es la relación que menos especificaciones semánticas requiere para llevarse a cabo; basta que entre una y otra unidad exista una temática compartida. Otra relación dentro de esta misma subcategoría, como la Lista, tampoco sugiere mayores sujeciones semánticas, sin embargo, su dificultad radica en las múltiples unidades nucleares que requiere su conformación.

En la Justificación, especulamos que entra en juego la habilidad pragmática preservada por el paciente. En las conversaciones, el acto de justificar conlleva cierto grado de disculpa, de salvaguardarse ante una acción o una declaración; usualmente, el paciente suele justificarse por olvidar o no ahondar en información que su interlocutor pide. En última instancia, no hay que olvidar que la Justificación forma parte de las relaciones que están enfocadas en obtener la credibilidad del interlocutor.

Contrariamente, el perfil de relaciones características de la conversación en pacientes cognitivamente sanos fue más preciso. Podemos decir que las relaciones retóricas más formuladas por esta población son: Concesión, Causa involuntaria, Solución, Resultado involuntario, Interpretación, Lista y Circunstancia $(95 \%$ de significación estadística). Con un nivel de significación más laxo, podrían incluirse las relaciones de Resumen, Fondo, Resultado voluntario, Elaboración y Evidencia (90\% de significación estadística). Particularmente, dos relaciones fueron estadísticamente significativas independientemente del número de relaciones de nuestra muestra: Solución y Causa involuntaria.

Un supuesto consistente con el reporte de Abdalla et al. (2017), es que nuestros resultados señalan que la diferencia de producción de la Elaboración es significativa en la identificación de uno y otro grupo de adultos; más precisamente, dicha relación es 
característica de poblaciones sanas. La elaboración (véase ejemplo 10), es una relación que exige más información relacionada con el núcleo; dicho así, tiene sentido que ambas poblaciones la hayan formulado por encima de otras; el punto clave de su vinculación con adultos sanos se basa en que estos buscan dotar su discurso de detalles e información más precisa, objetivos que a un paciente con demencia le costaría cumplir.

En general, el resto de relaciones con correlación positiva poseen rasgos conceptuales importantes; por ejemplo, en su definición, el Resumen, la Interpretación e incluso la Solución, son relaciones que en su mayoría requieren la formulación de múltiples SDU y, por ende, un mayor número de relaciones (véase Figura 1). Para que el paciente con DTA sea capaz de formular una Solución o un Resumen, debe ejecutar sus funciones mnémicas (Lai, 2014; Szatloczki et al., 2015) y recordar las unidades semánticas nucleares anteriores para así direccionar su interpretación o formular una unidad global; por otro lado, la Interpretación es un proceso que requeriría de la habilidad pragmática de la inferencia, facultad que, se ha constatado, va disminuyendo en la población con demencia (Chapman et al., 1998).

La relación de Causa involuntaria y Resultado involuntario, por otro lado, presupone que el sujeto tenga conciencia de su nivel de acción ante un detonante o resultado, además de la capacidad de realizar inferencias (Spooren \& Sanders, 2008). En sujetos con DAT, existe una disyuntiva al analizar estas relaciones en su discurso: sopesar en qué medida influye la pérdida de empatía del paciente hacia su interlocutor, y con ello el discurso que construye entorno a sí mismo, o examinar qué tantas limitaciones impone el tipo de discurso al paciente en tanto narrador de temas autobiográficos, pues de esta índole son los tópicos discutidos en todas las conversaciones.

Por último, y como área exploratoria, perseguimos constatar que entre la interacción cuidador-paciente se desplegaban ciertas relaciones que tenían un fin pragmático y de apoyo alentadas por los primeros. No se llevó a cabo un mayor tratamiento estadístico de los datos debido a la muestra; convenimos en que haría falta, como tarea a futuro, una revisión de los mismos y un estudio más pormenorizado, tanto más cuanto que los interlocutores de los pacientes, sean familiares o expertos de la salud, deben tener en consideración todos los recursos pragmáticos y discursivos que puedan ayudarles a mejorar o tratar la comunicación con el enfermo.

Desde nuestra perspectiva, una vez más las relaciones halladas dicen mucho acerca del papel de los cuidadores en tanto moderadores de la conversación. El Fondo y la Motivación, por ejemplo, (véanse ejemplo 15 y Figura 2, respectivamente) son relaciones de presentación que suelen usarse para proporcionar más contexto y para animar o instar a la realización de algo. En pacientes que sufren afectaciones en la 
memoria reciente o presentan confusiones respecto al entorno o situación que los rodea, dichas relaciones resultan ser estrategias conversacionales para proporcionar contexto y antecedentes al paciente e instarlo así a proseguir con la conversación.

Las relaciones más utilizadas por los cuidadores, con mucho, son la Elaboración y la Reformulación. Es interesante que, mientras los cuidadores en conversaciones con adultos DAT se apoyaban más en la Elaboración, en los diálogos con adultos sanos utilizaban más estrategias de Reformulación. Dado que en preguntas directas (que no en argumentos) la reformulación es una relación de fácil seguimiento, sería de esperar que los cuidadores las produjeran de forma más inmediata en conversaciones con paciente enfermos.

De manera usual, se presentaban situaciones en donde, si el paciente no entendía la pregunta o la olvidaba, el cuidador también hacía uso de la Reformulación para ayudarlo a comprender mejor la pregunta. Esta también era la utilidad de la Elaboración: se aderezaba con información a la pregunta para que fuera correctamente comprendida (véase ejemplo 13).

Ciertamente, un análisis más a profundidad entre las relaciones emitidas por ambos hablantes en la conversación ayudaría a ver en qué grado, según la población adulta y el desempeño de cada paciente, los cuidadores les auxilian en la construcción conversacional. En general, haría falta un análisis pragmático que identificara de manera concisa las relaciones que resultan ser mejores estrategias para las necesidades de cada población.

\section{CONCLUSIONES}

El objetivo principal de este trabajo fue perfilar la producción de relaciones retóricas de poblaciones con la enfermedad de Alzheimer, poblaciones mentalmente sanas y sus cuidadores para obtener así una primera valoración de las mismas en tanto mecanismos de coherencia y estrategias pragmáticas que pudieran contribuir en un futuro al tratamiento de este tipo demencia.

Tentativamente, nuestros resultados señalan que un hablante cognitivamente sano formulará más relaciones retóricas y de más tipos que un paciente con demencia tipo Alzheimer. Es posible que el catálogo de relaciones del que se sirve cada población tenga que ver con las implicaciones tanto pragmáticas como semánticas que presupone el uso de una u otra.

Ahora, no es posible conocer las implicaciones del uso o desuso de una determinada relación, y por ende, la lógica de un discurso, si no nos detenemos en su implicación conceptual. No todas las relaciones conllevan las mismas habilidades semánticas o pragmáticas; existen relaciones cuya formulación es más simple que la emisión de otras, así como relaciones que, si bien son equiparables en su 
conceptualización, poseen diferencias sutiles entre sí. El grupo de relaciones utilizadas por la población sana, por ejemplo, denotan de procedimientos discursivos complejos: formulación de subgrupos, mantenimiento de tópicos, replanteamientos y valoraciones interpersonales.

Adicionalmente, observamos también que ciertas relaciones retóricas pueden fungir como determinados recursos pragmáticos durante la conversación. Estos datos son valiosos para las investigaciones que tienen como propósito contribuir al tratamiento logopédico de los adultos que ya padecen demencia.

Somos conscientes que el número de sujetos que conformaron ambas muestras no es elevado, ya que el corpus, Carolinas' Conversations Collection en Español, es un proyecto de reciente conformación, aunque en constante incremento. Sin embargo, se han obtenido datos con significación estadística que muestran resultados interesantes. Asimismo, pese a que algunas de las proposiciones obtenidas en este estudio no son contundentes según las pruebas estadísticas, tienen un valor potencial en tanto antecedentes para investigaciones futuras. En este sentido, el trabajo insta a continuar y profundizar en estudios de poblaciones hispanohablantes que ahonden o precisen, con ayuda de este enfoque teórico, en el tratamiento de este síndrome.

\section{REFERENCIAS BIBLIOGRÁFICAS}

Abdalla, M., Rudzicz, F. \& Hirst, G. (2017). Rhetorical structure and Alzheimer's disease. Aphasiology, 32(1), 41-60.

Ahmed, S., Haigh, A. H., Jager, C. A. \& Garrard P. (2013). Connected speech as a marker of disease progression in autopsy-proven Alzheimer's disease. Brain, 136, 3727-3737.

Albert Cabrera, M. J., Martínez Pérez, R., Gutiérrez Ravelo, A., Hakim Rodríguez, D. \& Pérez Davison, G. (2014). Patogenia y tratamientos actuales de la enfermedad de Alzheimer. Revista Cubana de Farmacia, 48(3), 508-518.

Asgari, M., Kaye, J. \& Dodge, H. (2017). Predicting mild cognitive impairment from spontaneous spoken utterances. Alzheimer's \& Dementia, 3(2), 219-228.

Boschi, V., Catricala, E., Consonni, M., Chesi, C., Moro, A. \& Cappa, S. F. (2017). Connected speech in neurodegenerative language disorders: A review. Frontiers in psychology, 8, 269.

Brandão, L., Lima, T. M., Parente, M. \& Peña-Casanova, J. (2017). Discourse coherence and its relation with cognition in Alzheimer's disease. Revista Psicologia em Pesquisa, 7(1), 99-107. 
Carlson, L., Marcu, D. \& Okurowski, M. E. (2003). Building a discourse-tagged corpus in the framework of rhetorical structure theory. En J. C. J. van Kuppevelt \& R. W. Smith (Eds.), Current and new directions in discourse and dialogue. Text, Speech and Language Technology (pp. 85-112). Dordrech: Springer.

Cera, M. L., Ortiz, K. Z., Bertolucci, P. H. F. \& Minett, T. (2018). Phonetic and phonological aspects of speech in Alzheimer's disease. Aphasiology, 32(1), 88102.

Chafe, W. (1994). Discourse, consciousness, and time. Chicago: University of Chicago Press.

Chapman, S. B., Highley, A. P. \& Thompson, J. L. (1998). Discourse in fluent aphasia and Alzheimer's disease: Linguistic and pragmatic considerations. Journal of Neurolinguistics, 11(1-2), 55-78.

Das, D. \& Taboada, M. (2014). RST signalling corpus annotation manual. Burnaby Canadá: Simon Fraser University.

Davis, B. H. \& Maclagan, M. (2009). Examining pauses in Alzheimer's discourse. American Journal of Alzhbeimer's Disease \& Other Dementias, 24(2), 141154.

de Lira, J. O., Ortiz, K. Z., Campanha, A. C., Bertolucci, P. H. F. \& Minett, T. S. C. (2011). Microlinguistic aspects of the oral narrative in patients with Alzheimer's disease. International Psychogeriatrics, 23(3), 404-412.

Dijkstra, K., Bourgeois, M. S., Allen, R. S. \& Burgio, L. D. (2004). Conversational coherence: Discourse analysis of older adults with and without dementia. Journal of Neurolinguistics, 17(4), 263-283.

Fraser, K. C., Meltzer, J. A. \& Rudzicz, F. (2016). Linguistic features identify Alzheimer's disease in narrative speech. Journal of Alzheimer's Disease, 49(2), 407-422.

Garayzábal-Heinze, E. (2009). La lingüística clínica: Teoría y práctica. En J. L. Jiménez \& L. Timofeeva (Eds.), Investigaciones Lingüisticas en el Siglo XXI (pp. 131-168). Universidad de Alicante: España.

García Meilán, J., Martínez-Sánchez, F. \& Ivanova, O. (2018). Speech production in persons with dementia. En J. García Meilán, F. Martínez-Sánchez \& O. Ivanova (Eds.), Current Alşheimer Research, 15(2), 102-103.

Garrard, P., Ralph, M. A. L., Watson, P. C., Powis, J., Patterson, K. \& Hodges, J. R. (2001). Longitudinal profiles of semantic impairment for living and nonliving concepts in dementia of Alzheimer's type. Journal of Cognitive Neuroscience, 13(7), 892-909. 
Garrido Medina, J. (2007). Relaciones de discurso. Pandora: Revue d'etudes hispaniques, 7 , 305-332.

Gayraud, F., Lee, H. R. \& Barkat-Defradas, M. (2011). Syntactic and lexical context of pauses and hesitations in the discourse of Alzheimer patients and healthy elderly subjects. Clinical Linguistics \& Phonetics, 25(3), 198-209.

Grasso, L., Díaz, M. C. \& Peraita, H. (2011). Deterioro de la memoria semánticoconceptual en pacientes con enfermedad de Alzheimer. Análisis cualitativo y cuantitativo de los rasgos semánticos producidos en una tarea verbal de definición categorial. Psicogeriatría, 3(4), 159-165.

Grimes, J. E., (1975). The thread of discourse (Vol. 207). Nueva York: Mouton Publishers.

Guendouzi, J. A. \& Müller, N. (2006). Approaches to discourse in dementia. Nueva York: Psychology Press.

Halliday, M. A. K. (1967). Notes on transitivity and theme in English: Part 2. Journal of Linguistics, 3(2), 199-244.

Hamilton, H. E. (1996). Intratextuality, intertextuality and the construction of identity as patient in Alzheimer's disease. Text-Interdisciplinary Journal for the Study of Discourse, 16(1), 61-90.

Hays, S. J., Niven, B., Godfrey, H. \& Linscott, R. (2010). Clinical assessment of pragmatic language impairment: A generalisability study of older people with Alzheimer's disease. Aphasiology, 18(8), 693-714.

Hernández-Domínguez, L., Ratte, S., Davis, B. \& Pope, C. (2016). Conversing with the elderly in Latin America: A new cohort for multimodal, multilingual longitudinal studies on aging. Ponenencia prentada en el 7th Workshop on Cognitive Aspects of Computational Language Learning. Berlín. Alemania.

Hernández-Domínguez, L., Ratté, S., Sierra-Martínez, G. \& Roche-Bergua, A. (2018). Computer-based evaluation of Alzheimer's disease and mild cognitive impairment patients during a picture description task. Alzheimer's \& Dementia, 10, 260-268.

Hoffmann, I., Nemeth, D., Dye, C. D., Pákáski, M., Irinyi, T. \& Kálmán, J. (2010). Temporal parameters of spontaneous speech in Alzheimer's disease. International Journal of Speech-Language Pathology, 12(1), 29-34.

Kavé, G. \& Goral, M. (2018). Word retrieval in connected speech in Alzheimer's disease: A review with meta-analyses. Aphasiology, 32(1), 4-26.

Kehler, A., Kertz, L., Rohde, H. \& Elman, J. L. (2008). Coherence and coreference revisited. Journal of Semantics, 25(1), 1-44. 
Kempler, D. (1995). Language changes in dementia of the Alzheimer type. Dementia and Communication, 98-114.

Kong, A. P. H., Linnik, A., Law, S. P. \& Shum, W. W. M. (2017). Measuring discourse coherence in anomic aphasia using Rhetorical Structure Theory. International journal of speech-language pathology, 20(4), 406-421.

Lai, Y. H. (2014). Discourse features of Chinese-speaking seniors with and without Alzheimer's disease. Language and Linguistics, 15(3), 411-434.

Laine, M., Laakso, M., Vuorinen, E. \& Rinne, J. (1998). Coherence and informativeness of discourse in two dementia types. Journal of Neurolinguistics, 11(1-2), 79-87.

Lascarides, A. \& Asher, N. (2008). Segmented discourse representation theory: Dynamic semantics with discourse structure. En H. Bunt \& R. Muskens (Eds.), Computing meaning (pp. 87-124). Dordrecht: Springer.

López-de-Ipiña, K., Alonso, J. B., Travieso, C. M., Solé-Casals, J., Egiraun, H., Faundez-Zanuy, M. \& Lizardui, U. M. D. (2013). On the selection of noninvasive methods based on speech analysis oriented to automatic Alzheimer disease diagnosis. Sensors, 13(5), 6730-6745.

Mann, W. C. \& Thompson, S. A. (1988). Rhetorical structure theory: Toward a functional theory of text organization. Text-Interdisciplinary Journal for the Study of Discourse, 8(3), 243-281.

Mann, W. C., Matthiessen, C. M. \& Thompson, S. A. (1992). Rhetorical structure theory and text analysis. Discourse description: Diverse linguistic analyses of a fundraising text, 39-78.

Martin, J. R. (2001). Cohesion and texture. En D. Schiffrin, D. Tannen \& H. Hamilton (Eds.), The Handbook of Discourse Analysis (pp. 35-53). Massachussets: Blackwell Publishers.

Meilán, J. J., Martinez-Sánchez, F., Carro, J., López, D. E., Millian-Morell, L. \& Arana, J. M. (2014). Speech in Alzheimer's disease: Can temporal and acoustic parameters discriminate dementia? Dementia and Geriatric Cognitive Disorders, 37(5-6), 327-334.

Meilán, J. J., Martínez-Sánchez, F., Carro, J., Sánchez, J. A. \& Pérez, E. (2012). Acoustic markers associated with impairment in language processing in Alzheimer's disease. The Spanish journal of psychology, 15(2), 487-494. 
Mueller, K. D., Hermann, B., Mecollari, J. \& Turkstra, L. S. (2018). Connected speech and language in mild cognitive impairment and Alzheimer's disease: A review of picture description tasks. Journal of clinical and experimental neuropsychology, 40(9), 917-939.

Nicholas, M., Obler, L. K., Albert, M. L. \& Helm-Estabrooks, N. (1985). Empty speech in Alzheimer's disease and fluent aphasia. Journal of Speech, Language, and Hearing Research, 28(3), 405-410.

Orange, J. B., Lubinski, R. B. \& Higginbotham, D. J. (1996). Conversational repair by individuals with dementia of the Alzheimer's type. Journal of Speech, Language, and Hearing Research, 39(4), 881-895.

Paulino, A. \& Sierra, G. (2017). Applying the rhetorical structure theory in alyheimer patients' speech. Ponencia presentada en el 6th Workshop on Recent Advances in RST and Related Formalisms. Santiago de Compostela, España.

Paulino, A., Sierra, G., Hernández-Domínguez, L., da Cunha, I. \& Bel-Enguix, G. (2018). Rhetorical relations in the speech of Alzheimer's patients and healthy elderly subjects: An approach from the RST. Computación y Sistemas, 22(3), 895905.

Pérez Mantero, L. (2014). Interacción y predictividad: Los intercambios conversacionales con hablantes con demencia tipo alzhéimer. Revista de Investigación Lingüistica, 17, 97-118.

Pérez Mantero, L. (2015). La transgresión de las máximas conversacionales en hablantes con demencia tipo Alzhéimer. E-Aesla. Revista Digital de Lingüística Aplicada, 1, 1-9.

Pietrosemoli, L. (1996). Coherencia y cohesión en el discurso afásico. Lengua y habla, 1(1), 78-88.

Pistono, A., Jucla, M., Barbeau, E. J., Saint-Aubert, L., Lemesle, B., Calvet, B. \& Pariente, J. (2016). Pauses during autobiographical discourse reflect episodic memory processes in early Alzheimer's disease. Journal of Alzheimer's Disease, 50(3), 687-698.

Polanyi, L. (1996). The linguistic structure of discourse. Países Bajos: Tilburg University.

Renkema, J. (2008). Relaciones discursivas y variables de conectividad. Revista Signos. Estudios de Lingüistica, 41(66), 65-80. 
Rentoumi, V., Raoufian, L. Ahmed, S., de Jager, C. \& Garrard, P. (2014). Features and machine learning classification of Connected Speech Samples from patients with Autopsy Proven Alzheimer's Disease with and without additional vascular pathology. Journal of Alzheimer's Disease, 42, 3-17.

Ripich, D. N., Carpenter, B. D. \& Ziol, E. W. (2000). Conversational cohesion patterns in men and women with Alzheimer's disease: A longitudinal study. International Journal of Language \& Communication Disorders, 35(1), 49-64.

Ripich, D. N. \& Terrell, B. Y. (1988). Patterns of discourse cohesion and coherence in Alzheimer's disease. Journal of Speech and Hearing Disorders, 53(1), 8-15.

Roa Rojas, P. A., Martínez Ruíz, A. \& García Peña, M. C. (2017). Marco Conceptual. En L. M. Gutiérrez Robledo, M. C. García Peña, P. A. Roa Rojas \& A. Martínez Ruíz (Eds.), La enfermedad de Alzheimer y otras demencias como problema nacional de salud (pp. 1-15). México: Intersistemas.

Rochon, E., Leonard, C. \& Goral, M. (2018). Speech and language production in Alzheimer's disease. Aphasiology, 32, 1-3.

Sajjadi, S. A., Patterson, K., Tomek, M. \& Nestor, P. J. (2012). Abnormalities of connected speech in semantic dementia vs Alzheimer's disease. Aphasiology, 26(6), 847-866.

Sanders, T. \& Maat, H. P. (2006). Cohesion and coherence: Linguistic approaches. En K. Brown, (Ed.), Encyclopedia of Language \& Linguistics (pp. 591-595). Ámsterdam: Elsevier.

Silagi, M. L., Bertolucci, P. H. F. \& Ortiz, K. Z. (2015). Naming ability in patients with mild to moderate Alzheimer's disease: What changes occur with the evolution of the disease? Clinics, 70(6), 423-428.

Slegers, A., Filiou, R. P., Montembeault, M. \& Brambati, S. M. (2018). Connected speech features from picture description in Alzheimer's disease: A systematic review. Journal of Alzheimer's Disease, (Preprint), 1-26.

Spooren, W. \& Sanders, T. (2008). The acquisition order of coherence relations: On cognitive complexity in discourse. Journal of pragmatics, 40(12), 2003-2026.

Stent, A. \& Allen, J. (2000). Annotating argumentation acts in spoken dialog. Nueva York: University of Rochester.

Szatloczki, G., Hoffmann, I., Vincze, V., Kálmán, J. \& Pakaski, M. (2015). Speaking in Alzheimer's disease, is that an early sign? Importance of changes in language abilities in Alzheimer's disease. Frontiers in Aging Neuroscience, 7, 1-7. 
Taboada, M. T. (2004). Building coherence and cohesion: Task-oriented dialogue in English and Spanish (Vol. 129). Filadelfia: John Benjamins.

Taboada, M. T. \& Mann, W. C. (2006). Rhetorical structure theory: Looking back and moving ahead. Discourse Studies, 8(3), 423-459.

Taler, V., Baum, S. R., Chertkow, H. \& Saumier, D. (2008). Comprehension of grammatical and emotional prosody is impaired in Alzheimer's disease. Neuropsychology, 22(2), 188.

Toledo, C. M., Aluísio, S. M., dos Santos, L. B., Brucki, S. M. D., Trés, E. S., de Oliveira, M. O. \& Mansur, L. L. (2018). Analysis of macrolinguistic aspects of narratives from individuals with Alzheimer's disease, mild cognitive impairment, and no cognitive impairment. Alzheimer's \& Dementia: Diagnosis, Assessment \& Disease Monitoring, 10, 31-40.

Tosto, G., Gasparini, M., Lenzi, G. L. \& Bruno, G. (2011). Prosodic impairment in Alzheimer's disease: Assessment and clinical relevance. The Journal of neuropsychiatry and clinical neurosciences, 23(2), 21-23.

Toth, L., Hoffmann, I., Gosztolya, G., Vincze, V., Szatloczki, G., Banreti, Z. \& Kálmán, J. (2018). A speech recognition-based solution for the automatic detection of mild cognitive impairment from spontaneous speech. Current Alzheimer Research, 15(2), 130-138.

Valles González, B. (2006). La cohesión y la coherencia en la conversación del paciente con demencia: Un estudio discursivo. Revista de la Asociación Colombiana de Gerontología y Geriatría, 20(1), 882-886.

Valles González, B. (2009). Intercambios comunicativos en la afasia y en la demencia: Un estudio comparativo del uso de las reparaciones conversacionales. Letras, 51(78), 249-273.

Van Dijk, T. A., (1998). Texto y contexto: Semántica y pragmática del discurso. Barcelona: Cátedra.

Van Lancker Sidtis, D., Choi, J., Alken, A. \& Sidtis, J. J. (2015). Formulaic language in Parkinson's disease and Alzheimer's disease: Complementary effects of subcortical and cortical dysfunction. Journal of Speech, Language \& Hearing Research, 58(5), 1493-1506.

Varela Suárez, A. (2018). The question-answer adjacency pair in dementia discourse. International Journal of Applied Linguistics, 28(1), 86-101.

Zhu, Z., Novikova, J. \& Rudzicz, F. (2018). Detecting cognitive impairments by agreeing on interpretations of linguistic features, $A R X I V, 1-8$. 


\section{AGRADECIMIENTOS}

Los autores agradecen a los proyectos CONACYT: "Fronteras de la ciencia y Ampliación del corpus multiétnico de personas con edad avanzada", al proyecto CONACYT [231979] y a la colaboración del Ministère des Relations internationales et de la Francophonie Quebec-CONACYT. También reconocemos al contrato Ramón y Cajal (RYC-2014-16935) del Departamento de Filologías Extranjeras y sus Lingüísticas de la Universidad Nacional de Educación a Distancia (UNED), con el apoyo de los grupos de investigación ACTUALing e IULATERM, y al Natural Sciences and Engineering Research Council of Canada [RGPIN-2018-05714]. Finalmente, agradecemos a la doctora Sylvie Ratté, principal responsable de la recolección de los datos utilizados y al Laboratoire d'ingenierie cognitive et Sémantique de'l École de Technologie Supérieure.

\section{NOTAS}

1 "Any text in spoken English is organized into what may be called "information unit" The distribution of the discourse into information units is obligatory in the sense that the text must consist of a sequence of such units [...] the speaker is free to decide where each information unit begins and ends and how it is organized internally [...] is realized phonologically by "tonality" the distribution of the text into tone groups: one information unit is realized as one tone group" (Halliday, 1967: 199-200).

${ }^{2}$ Chafe (1994) nombra intonation units a las unidades de procesamiento mental y lingüístico, cuyos límites son dados por cambio de frecuencia en la voz, en su duración, intensidad, silencios, cambios de voz o cambios de turno.

${ }^{3}$ La clasificación se encuentra disponible en la página oficial de la RST:

http://www.sfu.ca/rst/

${ }^{4}$ Disponible en: https://sourceforge.net/projects/trans/files/transcriber/1.5.1/ 